stellt und berücksichtigen auch stets das numerische und graphische praktische Rechnen. Die Sammlung ist allen Studierenden wärmstens zu empfehlen.

.H. Hornich.

\title{
A. Witting, Repetitorium und Aufgabensammlung zur Integralrechnung
} (Sammlung Göschen, 147), $117 \mathrm{~S}$. W. de Gruyter, Berlin 1934. Preis geb. RM 1,62.

Die einzelnen Kapitel enthalten nach einem knappen, aber exakten und dabei gut lesbaren theoretischen Teil immer gleich eine Fülle von (insgesamt 305) Aufgaben, die teils ausführlich durchgerechnet werden, teils nur mit der Lösung versehen sind. Besonders sind die Anwendungen auf die Mechanik und Elektrizität in den letzten beiden Abschnitten hervorzuheben. - Obwohl das Büchlein der Ergänzung zum Band: "Integralrechnung" desselben Verfassers und in derselben Sammlung dient, ist es doch selbständig zu lesen; auch sind die Aufgaben grundsätzlich andere. Man kann das Buch nur bestens empfehlen.

H. Hornich.

o. Th. Bürklen, Mathematische Formelsammlung, hrsg. von F. Ringleb, 3. Auflage (Sammlung Göschen, 51), $270 \mathrm{~S}$. W. de Gruyter, Berlin 1936. Preis geb. RM 1,62 .

Die beliebte Formelsammlung ist nun in dritter Auflage herausgekommen; gegenüber der früheren Auflage sind die Veränderungen nicht sebr bedeutend. Bei der analytischen Geometrie des Raumes wird jetzt auch die Vektorrechnung gebracht, jedoch so, daß neben der rektoriellen Behandlung auch die bisher gebrauchte Koordinatenrechnung bleibt, um den mit der Vektorrechnung nicht Vertrauten die Benützung nicht zu erschweren. Ein Kapitel ist jetzt auch der Funktionentheorie gewidmet worden. Die große Beliebtheit, deren sich die Formelsammlung bisher erfreute, wird sicher auch der neuen Auflage zuteil werden.

H. Hornich.

R. Nevanlinna, Eindeutige analytische Funktionen (Grundlehren der mathematischen Wissenschaften in Einzeldarstellungen, Bd. 46), VIII +353 S. J. Springer, Berlin 1936. Preis geb. RM 29,40.

Die Lehre von den eindeutigen analytischen Funktionen hat in den letzten Jahrzehnten seit dem klassisch gewordenen Satz von Picard eine gewaltige Entwicklung genommen und wenn heute auch noch keineswegs ein AbschluB erreicht worden ist, so wird man doch die nunmehr vorliegende zusammenfassende Darstellung dieses schönen Gebietes sehr begrüßen, um so mebr, als diese einen der Schöpfer dieser Theorie zum Verfasser hat. (Wir verdanken übrigens dem Verf. schon einmal eine, damals - 1929 - naturgemäß weit weniger umfassende Darstellung dieser Lehre.)

Der Rieman $n$ sche Satz besagt bekanntlich, dab man eine beliebige, einfach zusammenhängende Ri e man n sche Fläche $G_{w}$ stets auf eine der drei Normalgebiete $G_{*}$ eineindeutig und konform abbilden kann: a) volle Ebene (elliptischer Fall), b) punktierte Ebene (parabolischer Fall), c) Einheitskreis (hyperbolischer Fall). Die Abbildungsfunktion $z(w)$ ist also eine einwertige, die Umkehrfunktion $w(z)$ eine eindeutige Funktion. Die Wertverteilungslehre untersucht nun die Verteilung der Stellen $z$, für welche $w(z)$ gleich einem vorgegebenen Wert $a$ ist. Zunächst lieferte diese Theorie (Hadamard, Borel, J ulia) weitgehende Verallgemeinerungen des Picardschen Satzes; darüber hinaus erwuchs nun, vornehmlich von den beiden Nevanlinna begründet, die hier dargestellte Theorie, die, auf neuen potentialtheoretischen Hilfsmitteln aufbauend, in den zwei Hauptsätzen gipfelt; die asymptotischen Eigenschaften der Funktion $w(z)$ werden nun in Verbindung gebracht zu den Verzweigungseigenschaften der Riemannschen Fläche $G_{w}$ und der Verzerrung durch die Abbildung $w(z)$, ein Zusammenhang, der bei der früheren Wertverteilungslehre fast gar nicht bestanden hatte. Die starke Hervorkehrung der geometrischen Gesichtspunkte bildet dabei einen besonderen Vorzug des Buches.

Das zentrale Problem, das bisher ungelöst blieb, ist die Frage, wie aus der topologisch-metrischen Struktur der Riemannschen Fläche $G_{w}$ ihre Zugehörigkeit zum parabolischen oder hyperbolischen Typus gefolgert werden kann. Die Sätze der Wertverteilungslehre liefern im allgemeinen nur gewisse notwendige Bedingungen für den parabolischen Typus, so daß das Typenproblem nur für wenige, ganz 